\title{
Slimy partners: the mucus barrier and gut microbiome in ulcerative colitis
}

\author{
Jian Fang ${ }^{1,2}$, Hui Wang ${ }^{3}$, Yuping Zhou ${ }^{4}$, Hui Zhang ${ }^{1}$, Huiting Zhou ${ }^{1}$ and Xiaohong Zhang ${ }^{1}$
}

\begin{abstract}
Ulcerative colitis (UC) is a chronic recurrent intestinal inflammatory disease characterized by high incidence and young onset age. Recently, there have been some interesting findings in the pathogenesis of UC. The mucus barrier, which is composed of a mucin complex rich in O-glycosylation, not only provides nutrients and habitat for intestinal microbes but also orchestrates the taming of germs. In turn, the gut microbiota modulates the production and secretion of mucins and stratification of the mucus layers. Active bidirectional communication between the microbiota and its 'slimy' partner, the mucus barrier, seems to be a continually performed concerto, maintaining homeostasis of the gut ecological microenvironment. Any abnormalities may induce a disorder in the gut community, thereby causing inflammatory damage. Our review mainly focuses on the complicated communication between the mucus barrier and gut microbiome to explore a promising new avenue for UC therapy.
\end{abstract}

\section{Introduction}

In recent years, the incidence of ulcerative colitis (UC), an inflammatory bowel disease (IBD) of unknown etiology, has been increasing globally, especially in some newly industrialized countries, including India and China ${ }^{1}$. Microbial infections such as those by Clostridium difficile have been described as a mono-associated cause of UC flare-ups ${ }^{2}$; however, there is growing evidence that UC is an overly robust mucosal immune response to dysbiosis of particular gut flora that is characterized by abnormal microbiota composition and bacterial products ${ }^{3,4}$. A balanced microbiome community is vital for maintaining mucus barrier homeostasis, which involves a dynamic balance of production, secretion, expansion, and proteolysis of mucus components. Commensal bacteria and their fermentation products (short-chain fatty acids, SCFAs) are implicated in the regulation of the production and secretion of mucin 2 (Muc2), the major component of

\footnotetext{
Correspondence: Xiaohong Zhang (zhangxiaohong1@nbu.edu.cn)

${ }^{1}$ Department of Preventive Medicine, Zhejiang Key Laboratory of

Pathophysiology, School of Medicine, Ningbo University, 818 Fenghua Road, Ningbo, Zhejiang, People's Republic of China

${ }^{2}$ College of Medicine, Shaoxing University, 508 Huancheng Road, Shaoxing,

Zhejiang Province, People's Republic of China
}

Full list of author information is available at the end of the article mucus, in sentinel goblet cells (sGCs) at crypt opening ${ }^{5}$ (Fig. 1a). The gut microbiome also influences the mucosal structure. Carbon dioxide $\left(\mathrm{CO}_{2}\right)$ generated from $\beta$-oxidation of SCFAs in colonocytes is converted by carbonic anhydrase to bicarbonate $\left(\mathrm{HCO}_{3}{ }^{-}\right)$, which in turn dictates the stratification of the mucus layers, such as the unfolding of mucin and resultant inner-towards-outer conversion of the mucus layer ${ }^{6}$ (Fig. 1b).

While the secreted, attached, hydrated, and stratified mucus barrier is mostly considered a simple lubricant layer overlying the epithelium, it also provides an environment for bacterial colonization and nourishes the commensal microbiota, thereby stabilizing the microbial community and promoting symbiotic interactions, resulting in microbial commensalism ${ }^{7}$. Mucus barrier abnormalities, including depleted upper crypt GCs, bacterial penetration of the inner mucus layer, and decreased core mucus components, such as FCGBP (human IgGFc binding protein), CLCA1 (calcium-activated chloride channel regulator 1), and ZG16 (zymogen granule protein $16)$, in active UC support the notion that an impaired mucus barrier may occur prior to the onset of inflammation in the pathogenesis of $\mathrm{UC}^{8}$. Environmental factors such as diet and lifestyle factors may shape the human gut 
a

Homeostasis

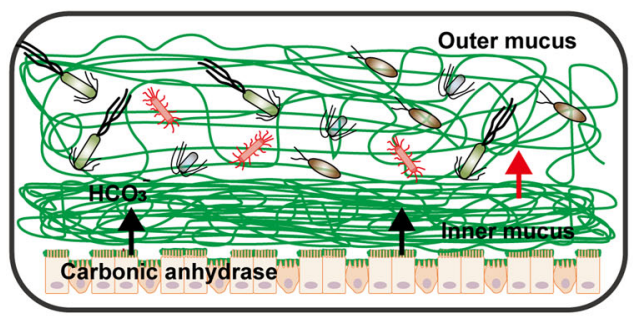

b

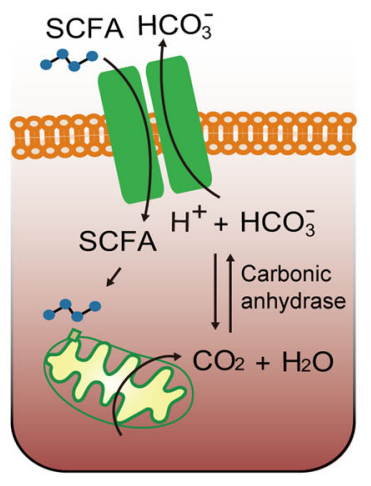

C

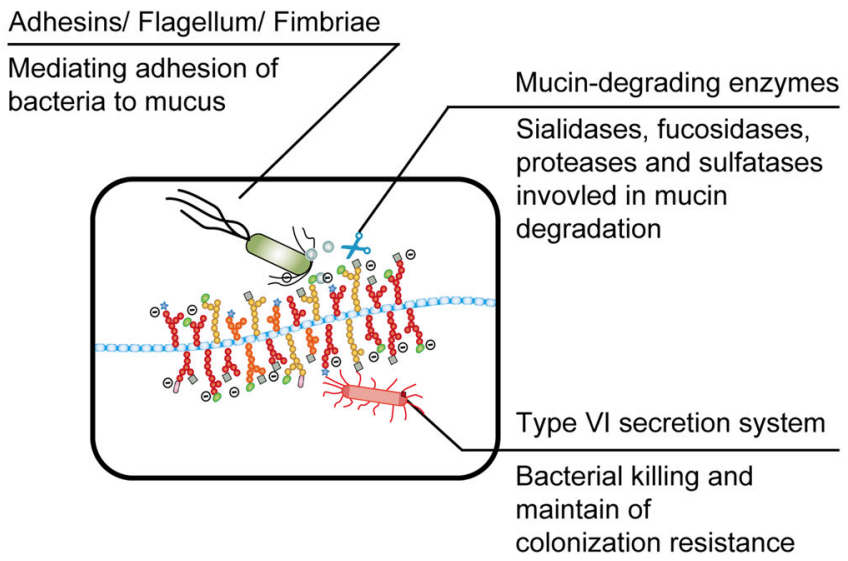

Fig. 1 The gut microbiome acts as orchestrator of the mucus barrier. a During homeostasis, the gut microbiome at the outer mucus layer modulates mucin production and secretion and mucus stratification mediated by $\mathrm{HCO}_{3}{ }^{-}$to maintain mucus barrier integrity. Dysbiosis induces impairment of the mucus barrier, accompanied by increased epithelium damage, bacterial translocation, goblet cell depletion, and host inflammation. b Gut microbiome-generated short-chain fatty acids enter colonocytes and are oxidized to generate $\mathrm{CO}_{2}$ that can be converted by carbonic anhydrase into $\mathrm{HCO}_{3}{ }^{-}$, which is the ideal physiological solution for precipitating calcium and raising the pH at the epithelial surface. This in turn promotes the stratification of the mucus layer. c Intestinal bacteria have evolved several strategies to adhere to the mucus barrier, including the use of adhesins, flagella, and fimbriae; achieve cross-feeding by mucin degradation; and maintain colonization resistance by means of a commensal type VI secretion system.

microbiome composition, thereby influencing mucus homeostasis and the development of intestinal inflammatory lesions 9 . Dietary fiber-deprived intestinal microbiota consume components of the mucus layer, leading to intestinal barrier dysfunction and increased susceptibility to pathogens and colitis occurrence ${ }^{10}$. It is obvious that the interplay between the microbiota and its 'slimy' partner, the mucus barrier, in the gut is constitutive. Therefore, any attempt to simply explore the underlying mechanism of UC from any single part of the biosystem (the mucus barrier and gut microbiota) is unwise. Currently, the development of microbiome-targeted therapeutic strategies for mild to moderate UC is growing ${ }^{11}$, and mucus barrier-associated colonization resistance involves commensal bacteria out-competing foreign microbes for space, trophic resources and bactericidal factors in the mucus barrier and decreasing the efficacy of fecal microbiome transplantation (FMT) therapy. This review provides insight into mucus barrier-gut microbiome interactions.

\section{The gut microbiome: orchestrator of the mucus barrier}

The gut microbiome adheres to mucus

Compared with the small intestine, the colonic epithelium is covered by mucus layers composed of a firm inner layer and loose outer layer that function to separate microbes from epithelial cells and provide a diffusion barrier to maintain a balanced community. The outer mucus layer is colonized with an abundance of commensal microbes, while the inner layer is relatively sterile (Fig. 1a). The combination of the mucus barrier and gut microbiome, composed of approximately 100 trillion symbiotic microbial cells and more than 9000 carbohydrate-degrading enzymes, is described as "the last human body organ"12. Commensal bacteria and 
pathogens have evolved several strategies to occupy a narrowly defined niche within the mucus barrier.

The first strategy is to adhere to the mucus by surface display of adhesins and extracellular appendages (fimbria) that bind to specific mucin glycans (Fig. 1c). Mucusbinding proteins (MUBs) are one class of effectors involved in the adherence of lactobacilli, abundant commensal bacteria in the human gut and the best studied example of mucus adhesins that confine commensal/ probiotic bacteria to the outer mucus layer ${ }^{13}$. Phylogenetically, adhesins are proteins characterized by the MUB domain, which shares homology with the Pfam-MucBP (mucin-binding protein) domains ${ }^{14}$. MUB and MucBP domain-containing proteins contain a $\mathrm{C}$-terminal recognition motif (LPxTG) that is recognized by a family of enzymes called sortases for covalent attachment to peptidoglycan of the bacterial cell wall and an N-terminal region for protein secretion, in addition to a signal peptide (Table 1). A number of proteins containing MUB homologs and MucBP domains have been found; for instance, the mucin/mucin-binding protein of Lactobacillus fermentum BCS87 (32-Mmubp), S-layer protein in L. acidophilus (SlpA), MucBP-containing mannose-specific adhesin (Msa), and elongation factor $\mathrm{Tu}(\mathrm{EF}-\mathrm{Tu})$ are highly prevalent in lactobacilli naturally existing in intestinal niches. Competitive adhesion studies have shown that MUB interacts with specific mucooligosaccharides and that MUB binding has little to no host specificity regarding mucus components ${ }^{15}$. The second strategy of mucus adhesion is mediated by fimbrial adhesion of commensal bacteria. For example, Escherichia coli, a commensal bacterium residing in the human gut, has the potential to act as an opportunistic pathogen. $E$. coli strains use extracellular fimbriae, which have a twodomain organization: lectin at the most external $\mathrm{N}$ terminal domain and pilin at the $\mathrm{C}$-terminus connecting to the rest of the fimbria. The affinity and specificity of the adhesion by fimbrial proteins are governed by recognition of mucus glycan epitopes, which are age-, organ-, and species-specific ${ }^{16}$. However, for many bacterial pathogens, binding to mucus is a crucial step in their colonization. Flagella, composed of flagellin arranged in helical chains, are an important evolved strategy for mucus adhesion during infection by some pathogens, and they play a critical role in biofilm formation ${ }^{17}$. Enterotoxigenic E. coli (ETEC) strains are major causes of morbidity and mortality due to diarrheal illness in developing countries. ETEC-secreted pathovar-specific proteins (such as EtpA, a two-partner adhesin conserved within the ETEC pathovar) can interact with both the tips of ETEC flagella and mucus glycans to form molecular bridges promoting bacterial adhesion and intestinal colonization of pathogens $^{18}$. Flagella are used as virulence factors by many enteropathogenic bacteria (e.g., Listeria monocytogenes,
Vibrio cholerae, E. coli, and Salmonella typhimurium) to traverse the mucus barrier, resulting in infection. Flagelladriven motility propels pathogens towards the epithelium and accelerates disease progression ${ }^{19}$. Many human pathogens, including C. difficile, pathogenic E. coli, Neisseria meningitidis, and Streptococcus pneumoniae, also employ phase-variable flagella and fimbriae to evade the host immune system and promote host colonization, persistence, motility, and virulence ${ }^{20}$.

\section{The gut microbiome feeds on mucin glycans}

After adhesion to mucins, colonization by colonic bacteria is initiated, while the degradation of diverse and structurally complex mucin glycans depends on the cooperative action of sialidases, sulfatases, proteases, and glycoside hydrolases (GHs) encoded by the genomes of mucin-degrading bacteria (Fig. 1c). Mucin-degrading carbohydrate-active enzyme (CAZyme) families include sialidases (GH33), fucosidases (GH29, GH95), bloodgroup endo- $\beta-1,4$-galactosidases (GH98), mucin core GHs (GH101, GH129, GH84, GH85, and GH89), and sulfatases (GH20, GH2, GH42, unclassified) ${ }^{21}$ (Table 2). Carbohydrate-binding modules (CBMs) in CAZymes mediate their adherence to carbohydrate substrates in mucin polymers ${ }^{22}$.

The adult gut microbiome consists of hundreds to thousands of different species of bacteria, with two predominant bacterial phyla: gram-positive Firmicutes and gram-negative Bacteroidetes ${ }^{23}$. Bacteroides spp. are prominent members of this microbial ecosystem and widely studied commensal bacteria ${ }^{24}$. They degrade a vast range of dietary and endogenous glycans by utilizing a complex transenvelope machinery known as starch utilization system (Sus)-like systems, which are encoded by coregulated clusters of genes known as polysaccharide utilization loci (PULs) $)^{25}$. Bacteroides spp., in particular $B$. thetaiotaomicron containing PULs, encode highly specific CAZymes and degrade a wide range of glycan substrates, thereby stratifying the niche space with different orders of substrate preferences, which is why they are sometimes referred to as "generalists" ${ }^{26}$. Akkermansia muciniphila can hydrolyze up to $85 \%$ of mucin structures using different enzyme combinations ${ }^{27}$, strengthen intestinal epithelial integrity, and fortify damaged gut barriers ${ }^{28}$. Interestingly, the abundances of $A$. muciniphila in both fecal samples and mucosal biopsies of UC patients are markedly reduced ${ }^{29}$. Butyrate, an SCFA produced by commensal bacteria, is the main energy source of colonocytes and exerts various beneficial effects, such as enhancement of intestinal barrier function. The production of butyrate using complex mucin glycans as a substrate is generally restricted to some Clostridium clusters (IV and XIVa) from the Firmicutes phylum. In addition, the butyrogenic effect of $A$. muciniphila ${ }^{30}$ is related to its 


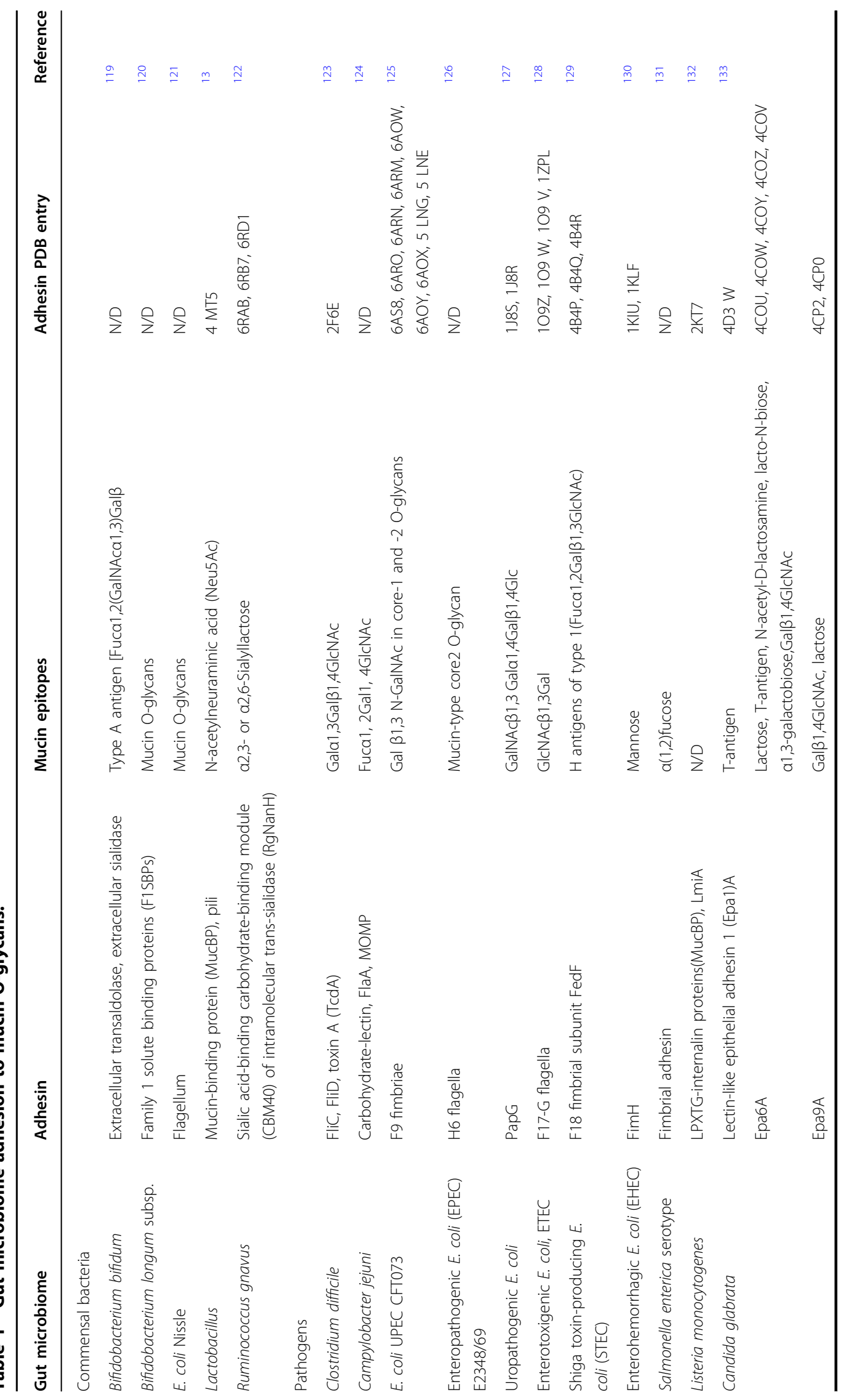




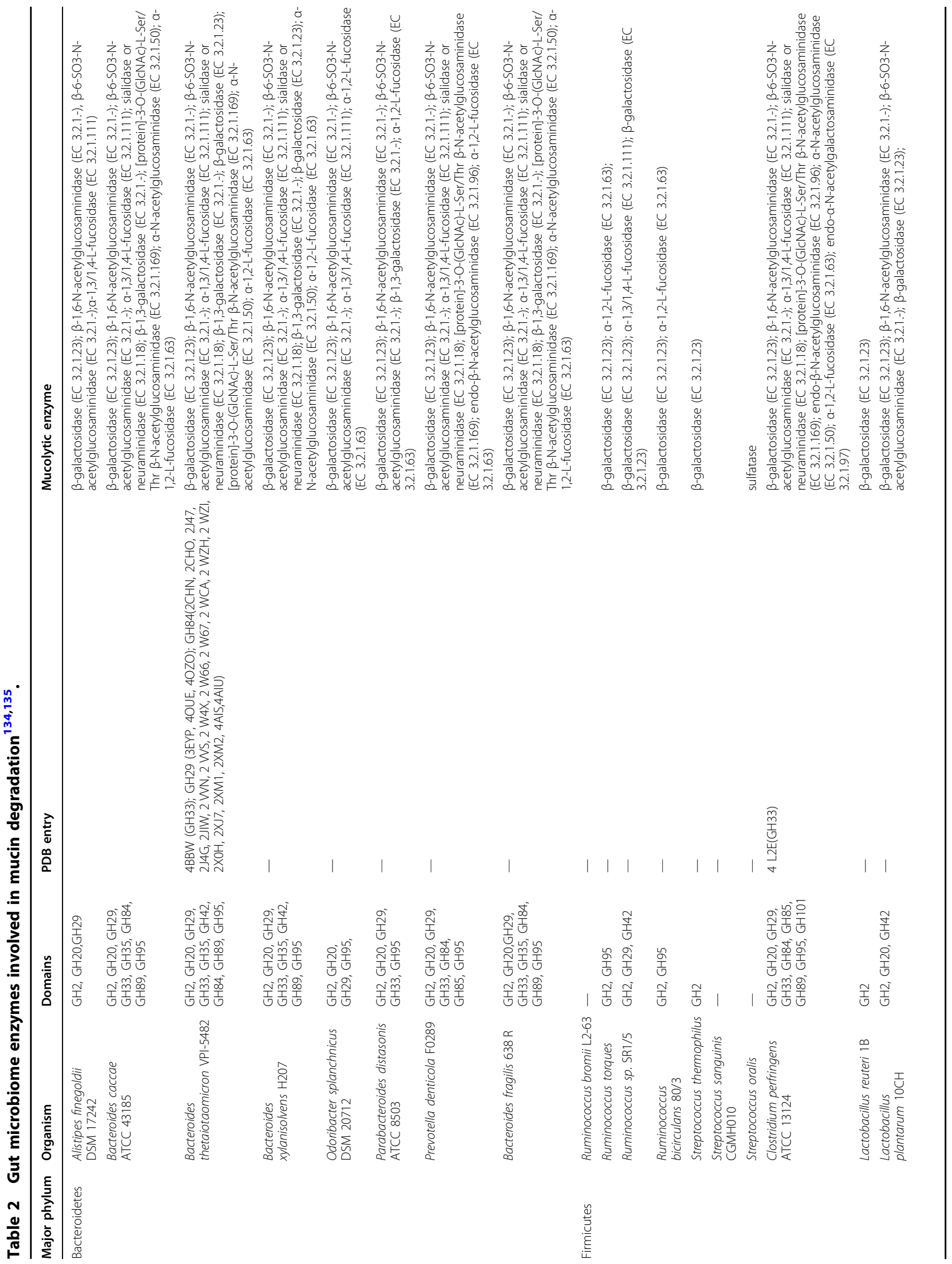




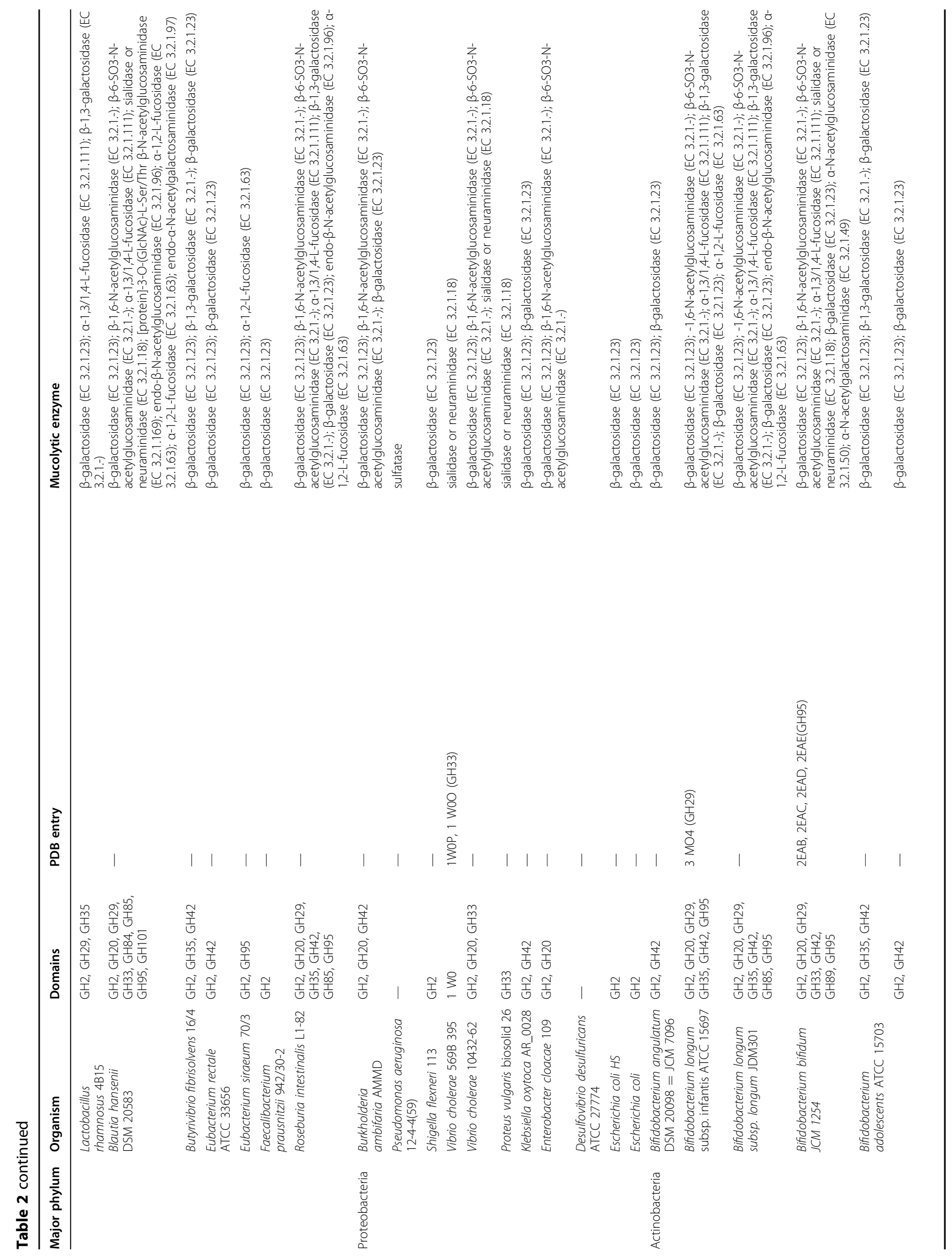




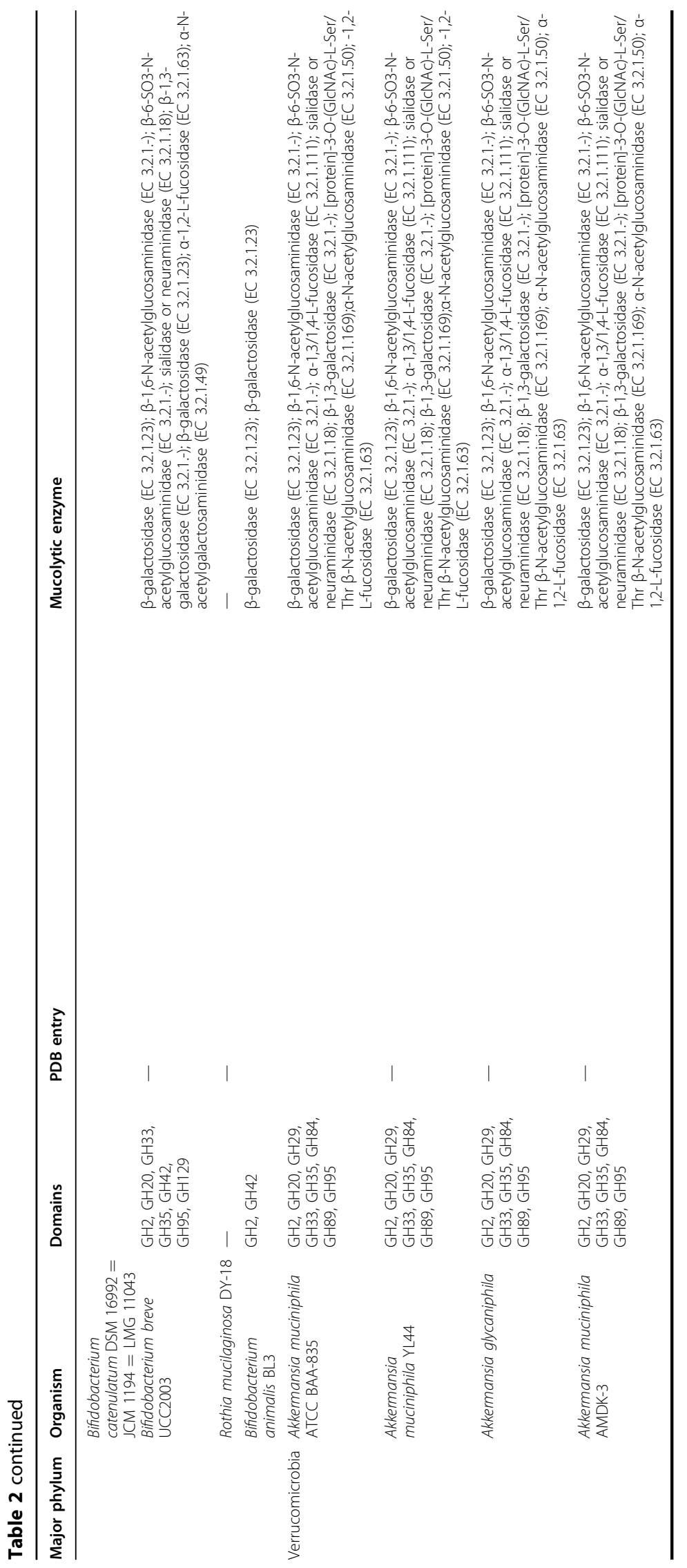


cross-feeding with mucus-degrading Clostridium clusters (IV and XIVa).

Notably, continual glycan degradation mediated by bacterial glycosidases may cause the disappearance of host-specific glycan epitopes and degradation of the protein backbone (Table 1). Dietary fiber-deprived intestinal microbiota have been shown to actively forage on the mucus layer, leading to dysfunction of the intestinal barrier and increased host susceptibility to pathogens and inflammation ${ }^{10}$ (Fig. 1c). It was reported that pathogenic Proteobacteria and Firmicutes species, including Salmonella enterica serovar Typhimurium, E. coli, and C. difficile, can benefit from cross-feeding through consumption of sialic acids from mucin molecules released by B. thetaiotaomicron. The expansion of pathogens during colitis is directly dependent on sialic acid released from host glycans catalyzed by sialidase ${ }^{31}$. Oral administration of a sialidase inhibitor and low levels of intestinal $\alpha 2,3-$ linked sialic acid decreased E. coli outgrowth and colitis severity in mice ${ }^{32}$. The cleavage site of the zinc metalloprotease zmpB from C. perfringens was established to be next to the mucus glycoprotein backbone (Ser and/or Thr residues), with optimal splicing of GlcNAc $\beta 1-3$ (Neu5Ac $\alpha 2-6)$, GalNAc $\alpha 1$, or GalNAc $\alpha 1$ ( $\alpha 2,6$-sialylated core 1 or core-3 O-glycan) ${ }^{33}$.

\section{The gut microbiome modulates mucus layer dynamics}

Mucin production was reported to be induced by the gut microbiome. SCFAs such as acetate, propionate, and butyrate, the fermentation products of commensal bacteria, enhance the synthesis of mucin and stimulated mucin secretion in mice ${ }^{34}$. Moreover, the stimulating effect of butyrate on $M u c 2$ expression is mediated via AP1 at the $M u c 2$ promoter $^{35}$. Lactic acid-based probiotics, containing Lactobacilli and Bifidobacteria, increase mucin production in human intestinal epithelial cells and block enteropathogenic $E$. coli invasion and adherence in vitro $^{36}$. Bifidobacterium species colonizing the intestinal mucus barrier modulate mucus production and expulsion by increasing the expression of GC markers such as Krüppel-like factor 4 (KLF4), trefoil factor 3 (TFF3), resistin-like molecule-beta (Relm- $\beta$ ), and Muc $2^{37}$. A randomized, placebo-controlled trial tested the efficacy and safety of a highly concentrated mixture of probiotic bacterial strains (VSL\#3) in active UC and its role in the maintenance of UC remission ${ }^{38}$ and demonstrated that the protective effect of VSL\#3 was related to enhanced colonic mucin expression and secretion in vivo and in vitro ${ }^{39}$. Several bacterial Toll-like receptor (TLR) ligands or effectors (e.g., lipopolysaccharide (LPS), flagellin, probiotic agents, commensal bacteria, and bacterial fermentation products) have been shown to trigger $M u c 2$ expression in colonic $\mathrm{sGCs}^{5,40}$. In addition, Muc2 production can also be enhanced by several stimuli, including
T-helper type 1 (Th1)- and Th2 cell-mediated cytokines, acute phase responses (colonic ischemia), and viral infection $^{41,42}$.

The gut microbiome is also involved in the modulation of mucus secretion by GCs. Non-O-glycosylated mucins with molecular weights of approximately $500 \mathrm{kDa}$ are synthesized in the endoplasmic reticulum of GCs and dimerized via disulfide bonds between the cystine knot (CK) domains. Mucin dimers transported to the Golgi apparatus are subjected to O-glycosylation and then multimerization by disulfide bonds at $\mathrm{N}$-terminal von Willebrand factor type D3 (vWF D3) domains. The resulting polymers reach molecular weights of up to 2.5 million $\mathrm{Da}^{43}$. Mucin multimers of 10-50 MDa [extended rods $1-10 \mu \mathrm{m}$ in length] are then packaged in an ordered state within secretory vesicles $(<1 \mu \mathrm{m})$ in the presence of low $\mathrm{pH}$ and high calcium ${ }^{44}$ (Fig. 1a). Upon secretion, the densely packed mucins can expand $>1,000$-fold, resulting in the formation of enormous net-like polymeric sheets ${ }^{45}$. Secretion of mucin can occur in at least two ways: regulated vesicle secretion and compound exocytosis. During regulated vesicle secretion (also called regulated exocytosis), the membrane of a secretory vesicle fuses with the plasma membrane by mediating the actions of typical vesicle exocytosis components such as syntaxins, mammalian uncoordinated-18 (Munc-18), vesicle-associated membrane proteins (VAMP), and synaptosomeassociated proteins (SNAP), and this is a tightly controlled process most often triggered by calcium $^{46}$. In compound exocytosis, storage vesicles rapidly fuse with the GC membrane after fusion with each other and empty all thecal contents ${ }^{47}$. The inner mucus layer is continuously renewed by mucin secretion of the surface GCs, and renewal of the inner mucus layer is estimated to occur every $1-2 \mathrm{~h}$ in live murine distal colonic tissue ${ }^{48}$. In general, spontaneous mucus production occurs at a rate of $240 \mu \mathrm{m} / \mathrm{h}$ in humans and $100 \mu \mathrm{m} / \mathrm{h}$ in the mouse colon; thus, the colonic mucus is continuously renewed at an average of $5-10 \mathrm{~L}$ per day ${ }^{49}$. Recently, sGCs have been shown to endocytose bacteria-derived TLR agonists such as LPS, lipid A, and flagellin but not lipotechoic acid, bacterial DNA, muramyl dipeptide, or $\gamma$-D-glutamylmesodiaminopimelic acid and activate TLR- and MyD88dependent NOD-like receptor family pyrin domain containing 6 (NLRP6) signaling to facilitate the exocytosis of mucin and flush bacteria away from crypt openings ex vivo ${ }^{5}$.

Stratification of the mucus layer has been shown to be indirectly influenced by the gut microbiome (Fig. 1a). An increase in $\mathrm{pH}$ and removal of $\mathrm{N}$-terminally bound single calcium ions are necessary for the conversion of the inner firm mucus layer to the outer loose mucus layer, the socalled mucus layer stratification ${ }^{50}$. In general, colonocytes are mainly dependent on adenosine triphosphate 
produced by the $\beta$-oxidation of butyrate, a metabolite of the gut microbiome, which is accompanied by the generation of $\mathrm{CO}_{2}$ that can be converted by carbonic anhydrase into $\mathrm{HCO}_{3}{ }^{-51}$; this is the ideal physiological solution for precipitating calcium and raising the $\mathrm{pH}$ at the epithelial surface ${ }^{52}$. The absence of $\mathrm{HCO}_{3}{ }^{-}$at the intestinal epithelial surface or inhibition of $\mathrm{HCO}_{3}{ }^{-}$ transepithelial transport decreases the amounts and rates of stimulated mucus release in vitro and in vivo ${ }^{53}$. For instance, facultative anaerobic bacteria such as pathogenic E. coli and Salmonella expand and invade the surface epithelium, thereby subverting colonocyte metabolism from $\beta$-oxidation of SCFAs to anaerobic glycolysis to promote their own luminal growth in competition against the gut microbiota by increasing the luminal bioavailability of oxygen $\left(\mathrm{O}_{2}\right)$, lactate, and additional electron acceptors, including tetrathionate $\left(\mathrm{S}_{4} \mathrm{O}_{6}{ }^{2-}\right)$ and nitrate $\left(\mathrm{NO}_{3}{ }^{-}\right)^{51,54}$. The resultant decrease in $\mathrm{HCO}_{3}{ }^{-}$in the lumen creates a high- $\mathrm{H}^{+}$environment, enhancing the $\mathrm{Ca}^{2+}$-binding of mucin polymers and making them more adhesive to each other in condensed mucin granules ${ }^{55}$. As a result, the structure of mucus layers is impaired, and host susceptibility to pathogens and even UC incidence increases; therefore, UC was postulated to be an energydeficient disease resulting from a failure to utilize butyrate $^{56}$.

\section{The mucus barrier regulates bacterial colonization} The mucus layer creates a habitat for commensal bacterial colonization

Hosts have evolved multiple strategies to maintain homeostasis of the intestinal microbiota (Fig. 2a). The best strategy is a highly adaptable protective mucus barrier exhibiting a heterogeneous spatial structure that establishes a habitat for commensal bacteria (Fig. 2b). The mucus barrier is a natural defense at the interface between host tissue and the luminal microbial community. Muc2 is the basic component of mucus that is continuously secreted and replenished by GCs in the large intestine. In the endoplasmic reticulum, the amino-terminal vWF and carboxy-terminal cystine knot (CK) domains of Muc2 mediate disulfide crosslinking of mucins to build a much larger mucin fishnet comprising thousands of monomers ${ }^{57,58}$. Muc2 consists of multiple domains, including the PTS [proline (Pro), threonine (Thr), and serine (Ser)] domain, a hallmark of the mucin family that is composed of a variable number of tandem repeats (VNTRs) that allow for heavy O-glycosylation with great heterogeneity in the Golgi apparatus and a stretched, brush-like arrangement of mucin. Neutral or negatively charged sugars, including N-acetylgalactosamine (GalNAc), sulfated acetyl-D-glucosamine (GlcNAc), D-galactose (Gal), sulfated Gal, sialic acid (Neu5Ac), and fucose, are attached to the PTS domains under catalysis by glycosyltransferases in the Golgi apparatus. Ultimately, these glycans account for up to $80 \%$ of the total mucin mass ${ }^{21}$. Importantly, the vast repertoire of O-glycosylated epitopes derived from the peripheral terminus of mucins (such as sialic acid and fucose) creates a habitat for unique bacterial ecosystems that thrive in proximity to host tissue ${ }^{59,60}$. Species of Bacteroides, the most abundant genus of the human gut microbiome, have a unique class of polysaccharideutilizing loci that are referred to as commensal colonization factors (CCFs). Bacteroides fragilis can penetrate the colonic mucus and reside deep within crypt channels, whereas strains with CCF mutations are defective in crypt invasion $^{61}$. It is known that reestablishment and resilience are fundamental characteristics of the gut microbial community ${ }^{61,62}$. The recolonization of gut $B$. fragilis following microbiome disruption caused by Citrobacter rodentium infection or antibiotic treatment is also dependent on CCFs ${ }^{61,63}$. Sulfatase (BF3086) and glycosyl hydrolase (BF3134) were annotated as mucosal colonization factors in B. fragilis. BF3086 is also important for $B$. fragilis to metabolize host mucus O-glycans ${ }^{64}$. During colonic mucus colonization, B. fragilis upregulates the expression of a set of candidate colonization factors, including BF3086 and BF3134, while in-frame deletions of these factors reduce its colonization abilities, which are fully or partially recovered by transcomplementation of BF3134 or BF3086 $6^{64}$.

The inhibition of symbiotic bacterial colonization by pathogens is mediated by degradation of mucosal glycosylation and includes decreasing fucosylation and increasing the release of sialic acid, which promotes the outgrowth and colonization of pathogenic E. coli ${ }^{32}$. LPS induces an increase in the expression of microbial virulence genes, such as RtxA (K10953) and hemolysin III (K11068), which enhance intestinal colonization of pathogenic microbes in fucosyltransferase 2 (Fut2)-deficient mice ${ }^{65}$. Enterohemorrhagic E. coli (EHEC) encodes a two-component sensing system (FusKR) consisting of a histidine sensor kinase (FusK) and response regulator (FusR). During colonization, EHEC cleaves fucose from mucin, thereby activating the FusKR signaling cascade and increasing the expression of virulence genes ${ }^{66}$. It was observed that S. typhimurium had significantly increased expression of genes (nan, fuc, and pdu) that utilize host mucin monosaccharides such as sialic acid, fucose, and propanediol, the catabolite of fucose, in gnotobiotic mice colonized with sialidase-expressing B. thetaiotaomicron ${ }^{67}$. Furthermore, antibiotic-treated conventional mice exhibited a transient surge in free sialic acid liberated by the resident microbiota from host mucus, promoting the expansion of Salmonella and C. difficile expressing sialic acid catabolic signaling ${ }^{67}$. As a result, it was concluded that antibiotic-associated pathogens such as S. typhimurium and $C$. difficile catabolize fucose and sialic acid 

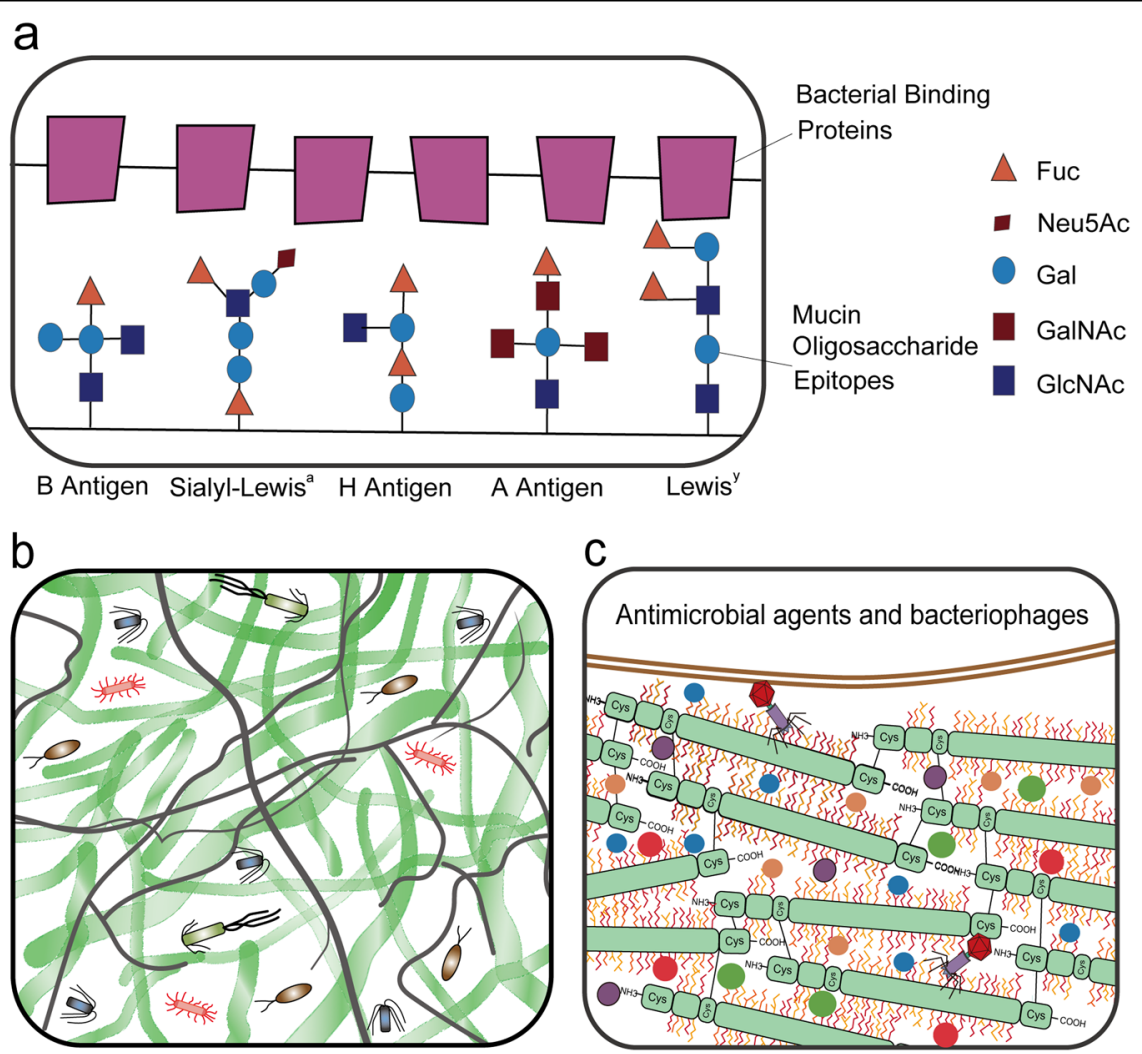

Fig. 2 The mucus barrier functions to modulate bacterial colonization. a The mucus barrier forms a fundamental niche for gut microbiome colonization, where the major O-glycan epitopes are sialic acid, fucose, N-acetylneuraminic acid (Neu5Ac), type A antigen [GalNAca1,3(Fuca1,2)Galß], and type $1 \mathrm{H}$ antigens [Fuca1,2Gal $\beta 1,3(\mathrm{GlcNAc})]$. b The mucus barrier dictates the spatial organization of microbes, forming a steric and orderly microorganism network to inhibit pathogen colonization. c The mucus barrier is also a scaffold containing antimicrobial agents [including RELM- $\beta$ (purple solid circle), ZG16 (blue solid circle), Ang4 (red solid circle), Lypd8 (green solid circle), slgA (orange solid circle), and bacteriophages] protecting epithelial cells against microbes.

liberated by the resident microbiota from mucin glycans in a resident microbiota-dependent manner ${ }^{67}$. Pathogens have also evolved a range of mucin-hydrolyzing enzymes called mucinases (glycosidases, proteases, and sulfatases) to degrade mucin complexes due to the mucus net-like nature. Notably, some commensal bacteria also produce mucinases, but their expression levels are much lower (Fig. 1c). Compared to pathogenic E. coli, commensal $E$. coli strains generate a lower amount of $\mathrm{YghJ}^{68}$, a lipoprotein with a zinc metalloprotease domain that is involved in mucin degradation as well as proinflammatory responses.

The colonization of commensals at the mucus layer also renders host resistance to pathogen colonization. CCFs mediate the production of a polysaccharide capsule around B. fragilis, thereby initiating an IL-36y response in mucosal macrophages of the gut to prevent colonization and infection by Klebsiella pneumoniae, which is a multidrug-resistant pathogen with high lethality ${ }^{69}$. Pathogens can be directly killed or inhibited by commensals that produce several antibacterial compounds.
For example, bacteriocins produced by commensal $E$. coli inhibit $\mathrm{EHEC}^{70}$, microbicides secreted by Enterobacteriaceae mediate interspecies competition in the inflamed gut $^{71}$, the bacteriocin thuricin produced by Bacillus thuringiensis inhibits the proliferation of $C$. difficile and $L$. monocytogenes ${ }^{72}$, and lantibiotics produced by lactic acid bacteria are used to target pathogens ${ }^{73}$. In addition, mucin was found to affect microbial behavior. For instance, gram-negative pathogens V. cholerae ${ }^{74}$ and S. Typhimurium $^{75}$ as well as commensals from the Bacteroides genus ${ }^{76}$ were reported to exert bactericidal effects mediated by the Type VI secretory system (T6SS) (Fig. 1c). It was recently revealed that mucin-associated glycans activate RetS, the sensor kinase of Pseudomonas aeruginosa, thereby inhibiting T6SS-dependent bacterial killing action ${ }^{77,78}$.

\section{Epithelial surface $\mathrm{pH}$ modulates the gut microbiota composition}

There are two key transport systems for $\mathrm{HCO}_{3}{ }^{-}$ extrusion into the colonic lumen: $\mathrm{Cl}^{-} / \mathrm{HCO}_{3}{ }^{-}$and SCFA/ $\mathrm{HCO}_{3}{ }^{-}$exchangers ${ }^{79}$ (Fig. 1b). Several lines of evidence 
indicate that $\mathrm{SCFA} / \mathrm{HCO}_{3}{ }^{-}$exchangers mediate ionized SCFA entry into colonocytes concomitant with an increase in luminal $\mathrm{pH}$ and a decrease in oxygen tension in both human and rodent colons ${ }^{80}$, which are vital for the stratification of the secreted mucin complex and colonization of obligate anaerobes, respectively. Treatment with live Bifidobacterium and its culture supernatants stimulated the expression of Slc26a3, a $\mathrm{Cl}^{-} / \mathrm{HCO}_{3}{ }^{-}$exchanger $^{81}$. Inflammation in the mid-distal ${ }^{82}$ or distal $\operatorname{colon}^{83}$ in Slc26a3-deficient mice was related to the loss of mucus secretion resulting from a remarkably low surface $\mathrm{pH}$ microclimate $^{83}$, a more aggressive microbiota ${ }^{82}$ and/or reduced microbiome diversity ${ }^{83}$. A luminal microenvironment with higher oxygen and lower $\mathrm{pH}$ could change the gut microbiota composition and drive an uncontrolled luminal expansion of $E$. coli and Salmonella ${ }^{84}$.

\section{Mucus viscosity determines the spatial organization of the gut microbiota}

The intestinal microflora is not evenly mixed but is spatially organized (Fig. 2c). Some mechanisms for the spatial organization of gut bacteria have been elucidated. Mucus is mainly composed of water $(95 \% \mathrm{w} / \mathrm{w})$, mucins $(0.2-5.0 \% \mathrm{w} / \mathrm{v})$, globular proteins $(0.5 \% \mathrm{w} / \mathrm{v})$, salts $(0.5-1.0 \% \mathrm{w} / \mathrm{w})$, lipids $(1-2 \% \mathrm{w} / \mathrm{w})$, DNA, cells, and cellular debris that form a dense, viscoelastic layer over epithelial cells ${ }^{85}$. There is a longitudinal (proximal to distal colon) viscosity gradient that increases progressively towards the distal colon in murine models, which restricts bacterial motility and confers spatial organization of bacterial populations. As a result, bacteria are selectively separated from the mucosa in the proximal colon and completely separated in the mid-distal colon ${ }^{86}$. Of note, uncovered cecum epithelium tips are a hotspot for $S$. typhimurium infection in mice due to the lack of a continuous mucus layer ${ }^{19}$. In the proximal murine colon, select bacterial populations intimately contact the mucosa and enter the crypts, thereby concentrating and forming a 20-240- $\mu \mathrm{m}$ thick film flanking the mucosa. The existence of vertical (surface to lumen) viscosity gradients within the colonic mucus layer was further demonstrated by low mucus viscosity at the crypt base and high viscosity at sites adjacent to the columnar epithelium or close to the intestinal lumen. A viscosity-dependent spatial distribution of bacteria in the murine colon revealed that short rods and cocci moved best in low viscosity, while long curly bacteria preferred a moderately viscous environment, and all bacteria were immobilized by high viscosity ${ }^{87}$. The lower viscosity of mucus at the crypt base makes intestinal cells more vulnerable to invasion by potential pathogens. In general, mucins contain several crosslinking domains to form dimers and larger-order structures via disulfide bonds that may be broken by sulfate-reducing bacteria (SRB), particularly Desulfovibrio desulfuricans $^{88}$. Many studies have described a high abundance of SRB detected in the mucosa of UC patients ${ }^{89,90}$. The resultant mucus barrier becomes less viscous and more permeable, allowing the gut microbiota in the gut lumen to interact with epithelial cells, thereby causing an aberrant immune response ${ }^{91}$. Recent studies have revealed the importance of site-specific gene expression for robust host-microbial symbiosis. B. fragilis near the epithelium upregulates the expression of genes involved in protein synthesis; moreover, compared to bacteria in the lumen, B. fragilis in mucus and tissue has high levels of sulfatase (BF3086) and glycosyl hydrolase $(\mathrm{BF} 3134)^{64}$. Intestinal mechanics are a host spatial control measure capable of regulating the abundance and persistence of gut bacteria. A $V$. cholerae symbiont native to zebrafish that governs its spatial organization using swimming motility and chemotaxis displayed strong localization to the foregut region, an anatomical region comparable to the mammalian small intestine with close contact with the intestinal epithelium to counter intestinal flow. In contrast, motility-deficient mutants that are susceptible to host spatial control largely aggregated within the intestinal mucus and were confined to the lumen, whereas chemotaxis-deficient mutants were restricted to the lumen of the midgut, and two mutants were susceptible to intestinal expulsion. Wild-type $V$. cholerae actively escapes mucus through regular changes in swimming direction mediated by chemotactic signaling ${ }^{92}$.

There are some factors influencing the viscosity of the mucus layer, TFF3 and $\mathrm{HCO}_{3}{ }^{-}$. TFF3, as a component of mucus, is essential for protection of the gastrointestinal mucosa $^{93}$. It is a small cysteine-rich acidic secreted protein that is covalently bound to the $\mathrm{C}$-terminal domain of Muc2 $^{94}$. Mucus viscosity has been shown to increase after the introduction of TFF3 dimers $(0.3 \% \mathrm{w} / \mathrm{v})$ compared with no treatment ${ }^{95}$. Tff3-knockout mice are more susceptible to dextran sulfate sodium (DSS)-induced colitis $^{96,97}$, while oral treatment with TFF3 protected against DSS-induced colitis in mice ${ }^{93}$.

There are two separate signaling pathways vital for normal mucus formation: $\mathrm{Ca}^{2+}$-mediated exocytosis of mucin granules of GCs and independent cAMP-mediated, cystic fibrosis transmembrane conductance regulator (CFTR)-dependent $\mathrm{HCO}_{3}{ }^{-}$secretion, which helps discharge sulfated and sialylated glycosylated domains $\mathrm{s}^{85}$ and stratifies exocytosed mucus ${ }^{98}$. Additionally, $\mathrm{HCO}_{3}{ }^{-}$also participates in mucin expansion and hydration mechanisms by reducing $\mathrm{Ca}^{2+}$ cross-linking in mucins, thereby decreasing the viscosity ${ }^{55}$. CFTR is the secretory chloride/ $\mathrm{HCO}_{3}{ }^{-}$channel; its dysfunction causes acidification of the mucus layer $(\mathrm{pH}<6.5)$ due to defective $\mathrm{HCO}_{3}{ }^{-}$release, resulting in increased mucus viscoelasticity and the formation of a stationary mucus layer in cystic fibrosis ${ }^{99}$. 


\section{The mucus barrier generates a protective shield}

Colonic mucus is a key component of the colonic barrier, as it is located at the interface between luminal microflora and the colonic mucosa. The mucus barrier effectively partitions the enteric epithelium from the microbiota as the first line of defense and supports the growth of intestinal commensals as an energy source. The development of colitis in animals lacking a functional mucus layer closely reflects clinical and cellular features in patients with active UC. Penetration of the inner mucus layer in the distal colon by pathogens and/or commensals often found in mice with colitis is related to impaired mucus barrier structure and function caused by genetic deficiency in Muc2 ${ }^{100}$, inactivation of glycosyltransferasemediated O-glycosylation of Muc2 ${ }^{101,102}$, deficiency of the NLRP6 inflammasome, or exposure to colitis-inducing chemicals ${ }^{103}$. Some pathogens such as enterohemorrhagic or enteropathogenic E. coli (EHEC or EPEC), C. rodentium, and $S$. typhimurium disrupt the protective mucus barrier, causing dysbiosis characterized by decreased abundances of Firmicutes and Verrucomicrobia and increased abundances of Bacteroidetes and facultative anaerobes $^{104}$, which adhere to or invade host epithelial cells beneath the mucus layer. The vicious cycle of dysbiosis and colonic inflammation is characterized by destruction of the mucus barrier and persistent overstimulation of the immune system by the microflora ${ }^{19}$. Chronic or intermittent dietary fiber deficiency pushes the resident microbiota to rely more heavily on endogenous nutrients (host-secreted mucin glycoproteins), leading to erosion of the colonic mucus barrier and exacerbation of colitis triggered by the mucosal pathogen $C$. rodentium ${ }^{10}$.

\section{Antimicrobial agents fortify the mucus barrier}

Importantly, the dense gel-forming structure of the mucus layer acts as a trap to stabilize numerous molecules, such as RELM- $\beta$ and zymogen granule protein 16 (ZG16), angiogenin 4 (Ang4), Ly6/PLAUR domain containing 8 (Lypd8), and secretory immunoglobulin A (sIgA) (Fig. 2b). RELM- $\beta$ exerts a microbicidal effect predominantly on gram-positive pathogens penetrating the mucus layer ${ }^{104}$. ZG16 prevents the adherence of bacteria to the epithelium by binding to the peptidoglycan of the bacterial cell wall ${ }^{105}$. Ang 4, another antimicrobial agent derived from GCs, is associated with Trichuris muris expulsion from the colonic epithelium of mice during inflammation ${ }^{106}$. B. thetaiotaomicron promotes Ang 4 expression, which inhibits the growth of some bacterial species, such as $L$. monocytogenes and Enterococcus faecalis ${ }^{107}$. Lypd 8, a highly glycosylated glycosylphosphatidylinositol-anchored protein selectively expressed in enterocytes, can bind to flagellated bacteria to inhibit bacterial invasion into the colonic epithelia when secreted into the lumen. Lypd8 strongly causes early-phase defense against $C$. rodentium, which can induce colitis by triggering attachment and effacement (A/E) lesions on colonic epithelia. Mechanistically, Lypd8 inhibits $C$. rodentium attachment to intestinal epithelial cells by binding to intimin, thereby protecting against enteric bacterial pathogens ${ }^{108}$. sIgA secreted as a dimer by colonocytes and integrated into the mucus layer exerts a critical function in trapping luminal bacteria to prevent unrestricted access of the microbiota to the epithelial surface ${ }^{109}$. The decreasing gradient of antimicrobial agents from the epithelial surface to the lumen is positively correlated with mucin concentration in the bilayered mucus matrix, which is why the intestinal mucus layers harbor significant antibacterial activity, whereas only low activity is detected in the luminal content. Because of the anti-inflammatory and antimicrobial nature of mucosal contents, the mucus layer generates a protective shield to prevent bacterial translocation and inappropriate immune stimulation of the epithelium ${ }^{110}$. However, when a functional mucus layer is absent, the gradient of antimicrobial agents is diminished, and the related defense system is eliminated from the intestine with fecal flow ${ }^{23}$.

\section{Bacteriophage attachment to mucus strengthens mucus defense}

Under homeostatic conditions, mucus provides protection against dysbiosis by bacteriophage deployment (Fig. 2b). Bacteriophage, a resident member of the gut microbiome, interacts with mucin glycoproteins in the mucus barrier though immunoglobulin-like domains that are exposed on the capsid, triggering nonhost-derived immunity, which is considered part of the innate immune system $^{111}$. Adherent invasive E. coli (AIEC) strain LF82 has type 1 pili mediating its binding to the host adhesion receptor carcinoembryonic antigen-related cell adhesion molecule 6 (CEACAM6), which is more strongly expressed in the ileal tissues of patients with Crohn's disease $(\mathrm{CD})^{112}$. A single day of oral treatment with a cocktail of bacteriophages was found to induce significantly decreased intestinal colonization by AIEC strain LF82 in CEABAC10 transgenic mice ${ }^{113}$. Moreover, this single dose of bacteriophage inhibited DSS-induced colitis symptoms over a two-week period in conventional mice colonized with LF82 ${ }^{114}$. Bacteriophage intervention is planned to be evaluated in patients with IBD in the United States ${ }^{113}$. Data from UC mouse models have revealed that some bacteriophages that infect bacteria with pathogenic potential (pathobionts) are elevated during colitis ${ }^{115}$. Specifically, an increased abundance of bacteriophages predicted to infect Streptococcus sp. and Alistipes and Clostridiales phages predicted to infect $C$. difficile were observed during colitis ${ }^{116}$. This elevated abundance of specific phages could be postulated as a proxy for strainlevel resolution of disease-causing bacteria during IBD $^{116}$. It has been reported that intestinal microbiota-associated 
phages attach to mucins and protect underlying epithelial cells from invading bacteria ${ }^{117}$. Spatial organization of the mucus generates a gradient of phage replication with lysogeny at the top mucosal layer and lytic predation in the bacteria-sparse intermediary layers ${ }^{117}$. However, animals with bacteriophage expansion, such as Caudovirales phages, exhibit a significant exacerbation of intestinal colitis $^{118}$. This inconsistency indicates a complex role of phages in IBD.

\section{Conclusion}

Massive advances in the etiology of UC over the past few decades have improved our understanding of the importance of active communication between the gut microbiota and the mucus barrier. It is evident that disturbance of this interplay is a vital pathological factor for UC development. From the perspective of intricate interactions between the mucus barrier and the gut microbiome in the gut microenvironment, it is important to explore interventional approaches to control inflammation or promote FMT. Hence, exploring promising therapeutic agents from the viewpoint of 'slimy' partners is necessary to effectively treat UC.

\section{Acknowledgements}

We would like to thank Editage (www.editage.cn) for English language editing. This study was supported by the National Natural Science Foundation of China (NSFC; Grant No. 81872620, 81673163), the Ningbo Natural Science Foundation (2018A610370), the Natural Science Foundation of Zhejiang Province (LY21H260001), the Zhejiang Key Laboratory of Pathophysiology (201802), the Zhejiang Public Welfare Technology Applied Research Project (LGF18H060006) and the Zhejiang Provincial Medical and Health Science and Technology plan (2018KY819). This study was sponsored by the K. C. Wong Magna Fund at Ningbo University, Shaoxing Municipal Science and Technology Project of Medical and Health (2020A13064) and the Scientific Research Projects of Shaoxing University (2019SK003).

\section{Author details \\ 'Department of Preventive Medicine, Zhejiang Key Laboratory of Pathophysiology, School of Medicine, Ningbo University, 818 Fenghua Road, Ningbo, Zhejiang, People's Republic of China. ${ }^{2}$ College of Medicine, Shaoxing University, 508 Huancheng Road, Shaoxing, Zhejiang Province, People's Republic of China. ${ }^{3}$ Department of Colorectal Surgery, Shaoxing people's Hospital, 568 North Zhongxing Road, Shaoxing, Zhejiang Province, People's Republic of China. ${ }^{4}$ The Affiliated Hospital of Medical School, Ningbo University, 247 Renmin Road, Ningbo, Zhejiang, People's Republic of China}

\section{Author contributions}

J.F., H.W., Y.Z. and H.Z. contributed equally to this work.

\section{Conflict of interest}

The authors declare no competing interests.

\section{Publisher's note}

Springer Nature remains neutral with regard to jurisdictional claims in published maps and institutional affiliations.

Received: 4 January 2021 Revised: 14 January 2021 Accepted: 31 January 2021.

Published online: 17 May 2021

\section{References}

1. Alatab, S. et al. The global, regional, and national burden of inflammatory bowel disease in 195 countries and territories, 1990-2017: a systematic analysis for the Global Burden of Disease Study 2017. Lancet Gastroenterol. Hepatol. 5, 17-30 (2020).

2. Clayton, E. M. et al. The vexed relationship between Clostridium difficile and inflammatory bowel disease: an assessment of carriage in an outpatient setting among patients in remission. Am. J. Gastroenterol. 104, 1162-1169 (2009).

3. Round, J. L. \& Mazmanian, S. K. The gut microbiota shapes intestinal immune responses during health and disease. Nat. Rev. Immunol. 9, 313-323 (2009).

4. Tremaroli, V. \& Bäckhed, F. Functional interactions between the gut microbiota and host metabolism. Nature 489, 242-249 (2012).

5. Birchenough, G. M., Nyström, E. E., Johansson, M. E. \& Hansson, G. C. A sentinel goblet cell guards the colonic crypt by triggering Nlrp6-dependent Muc2 secretion. Science 352, 1535-1542 (2016).

6. Gad, S. C. Toxicology of the gastrointestinal tract 2th edn. (CRC Press, Boca Raton, 2018).

7. Paone, P. \& Cani, P. D. Mucus barrier, mucins and gut microbiota: the expected slimy partners? Gut 69, 2232-2243 (2020).

8. Van der Post, S. et al. Structural weakening of the colonic mucus barrier is an early event in ulcerative colitis pathogenesis. Gut 68, 2142-2151 (2019).

9. Rothschild, D. et al. Environment dominates over host genetics in shaping human gut microbiota. Nature 555, 210-215 (2018).

10. Desai, M. S. et al. A dietary fiber-deprived gut microbiota degrades the colonic mucus barrier and enhances pathogen susceptibility. Cell 167, 1339-1353. e1321 (2016).

11. Costello, S. P. et al. Effect of fecal microbiota transplantation on 8-week remission in patients with ulcerative colitis: a randomized clinical trial. JAMA 321, 156-164 (2019).

12. Proctor, L. Priorities for the next 10 years of human microbiome research. Nature 569, 623-625 (2019).

13. Etzold, S. \& Juge, N. Structural insights into bacterial recognition of intestinal mucins. Curr. Opin. Struct. Biol. 28, 23-31 (2014).

14. Juge, N. Microbial adhesins to gastrointestinal mucus. Trends Microbiol. 20, 30-39 (2012).

15. Roos, S. \& Jonsson, H. A high-molecular-mass cell-surface protein from Lactobacillus reuteri 1063 adheres to mucus componentsThe GenBank accession number for the sequence reported in this paper is AF120104. Microbiology 148, 433-442 (2002).

16. Colomb, F. et al. Epithelial mucins and bacterial adhesion. in Carbohydr. Chem., Vol. 40 (ed. Amelia Pilar Rauter, A. P. R., Thisbe Lindhorst, Yves Queneau) 596-623 (Royal Society of Chemistry, 2014).

17. Lam, W. W.-L., Sun, K., Zhang, H. \& Au, S. W.-N. Crystal structure of flagellar export chaperone FliS in complex with flagellin and HP1076 of Helicobacter pylori. Front Microbiol. 11, 787 (2020).

18. Kumar, P. et al. Dynamic interactions of a conserved enterotoxigenic Escherichia coli adhesin with intestinal mucins govern epithelium engagement and toxin delivery. Infect. Immun. 84, 3608-3617 (2016).

19. Furter, M., Sellin, M. E., Hansson, G. C. \& Hardt, W.-D. Mucus architecture and near-surface swimming affect distinct Salmonella Typhimurium infection patterns along the murine intestinal tract. Cell Rep. 27, 2665-2678. e2663 (2019).

20. Garrett, E. M. et al. Phase variation of a signal transduction system controls Clostridioides difficile colony morphology, motility, and virulence. PLOS Biol. 17, e3000379 (2019).

21. Tailford, L. E., Crost, E. H., Kavanaugh, D. \& Juge, N. Mucin glycan foraging in the human gut microbiome. Front Genet. 6, 81 (2015).

22. Sidar, A. et al. Carbohydrate Binding Modules: Diversity of Domain Architecture in Amylases and Cellulases From Filamentous Microorganisms. Front Bioeng. Biotechnol. 8, 871-886 (2020).

23. Donaldson, G. P., Lee, S. M. \& Mazmanian, S. K. Gut biogeography of the bacterial microbiota. Nat. Rev. Microbiol. 14, 20-32 (2016).

24. Bloom, S. M. et al. Commensal bacteroides species induce colitis in hostgenotype-specific fashion in a mouse model of inflammatory bowel disease. Cell Host Microbe 9, 390-403 (2011).

25. Martens, E. C., Chiang, H. C. \& Gordon, J. I. Mucosal glycan foraging enhances fitness and transmission of a saccharolytic human gut bacterial symbiont. Cell Host Microbe 4, 447-457 (2008).

26. Walter, J. \& Ley, R. The human gut microbiome: ecology and recent evolutionary changes. Annu. Rev. Microbiol. 65, 411-429 (2011). 
27. Trastoy, B., Naegeli, A., Anso, I., Sjögren, J. \& Guerin, M. E. Structural basis of mammalian mucin processing by the human gut O-glycopeptidase OgpA from Akkermansia muciniphila. Nat. Commun. 11, 4844 (2020).

28. Reunanen, J. et al. Akkermansia muciniphila adheres to enterocytes and strengthens the integrity of the epithelial cell layer. Appl. Environ. Microbiol. 81, 3655-3662 (2015).

29. Png, C. W. et al. Mucolytic bacteria with increased prevalence in IBD mucosa augment in vitro utilization of mucin by other bacteria. Am. J. Gastroenterol. 105, 2420-2428 (2010).

30. Naito, Y., Uchiyama, K. \& Takagi, T. A next-generation beneficial microbe: Akkermansia muciniphila. J. Clin. Biochem. Nutr. 63, 33-35 (2018).

31. Sorbara, M. T. \& Pamer, E. G. Interbacterial mechanisms of colonization resistance and the strategies pathogens use to overcome them. Mucosal Immunol. 12, 1-9 (2019).

32. Huang, Y.-L., Chassard, C., Hausmann, M., Von Itzstein, M. \& Hennet, T. Sialic acid catabolism drives intestinal inflammation and microbial dysbiosis in mice. Nat. Commun. 6, 1-11 (2015).

33. Noach, I. et al. Recognition of protein-linked glycans as a determinant of peptidase activity. Proc. Natl Acad. Sci. USA 114, E679-E688 (2017).

34. Gaudier, E. et al. Butyrate specifically modulates MUC gene expression in intestinal epithelial goblet cells deprived of glucose. Am. J. Physiol. Gastrointest. Liver Physiol. 287, G1168-G1174 (2004).

35. Burger-van Paassen, $\mathrm{N}$. et al. The regulation of intestinal mucin MUC2 expression by short-chain fatty acids: implications for epithelial protection. Biochem. J. 420, 211-219 (2009).

36. Mack, D. R., Michail, S., Wei, S., McDougall, L. \& Hollingsworth, M. A. Probiotics inhibit enteropathogenic $E$. coli adherence in vitro by inducing intestinal mucin gene expression. Am. J. Physiol. Gastrointest. Liver Physiol. 276, G941-G950 (1999)

37. Engevik, M. A. et al. Bifidobacterium dentium fortifies the intestinal mucus layer via autophagy and calcium signaling pathways. mBio $\mathbf{1 0}$ e01087-01019 (2019)

38. Miele, E. et al. Effect of a probiotic preparation (VSL\# 3) on induction and maintenance of remission in children with ulcerative colitis. Am. J. Gastroenterol. 104, 437-443 (2009).

39. Caballero-Franco, C., Keller, K., De Simone, C. \& Chadee, K. The VSL\# 3 probiotic formula induces mucin gene expression and secretion in colonic epithelial cells. Am. J. Physiol. Gastrointest. Liver Physiol. 292, G315-G322 (2007).

40. Sicard, J.-F., Le Bihan, G., Vogeleer, P., Jacques, M. \& Harel, J. Interactions of intestinal bacteria with components of the intestinal mucus. Front Cell Infect. Microbiol. 7, 387 (2017).

41. Nyström, E. Colonic mucus structure and processing. Sweden: University of Gothenburg (2018)

42. Grootjans, J. et al. Ischaemia-induced mucus barrier loss and bacterial penetration are rapidly counteracted by increased goblet cell secretory activity in human and rat colon. Gut 62, 250-258 (2013).

43. Thornton, D. J., Rousseau, K. \& McGuckin, M. A. Structure and function of the polymeric mucins in airways mucus. Annu. Rev. Physiol. 70, 459-486 (2008).

44. Bansil, R. \& Turner, B. S. The biology of mucus: composition, synthesis and organization. Adv. Drug Deliv. Rev. 124, 3-15 (2018).

45. Ambort, D. et al. Calcium and $\mathrm{pH}$-dependent packing and release of the gelforming MUC2 mucin. Proc. Natl Acad. Sci. USA 109, 5645-5650 (2012).

46. Birchenough, G. M., Johansson, M. E., Gustafsson, J. K., Bergström, J. H. \& Hansson, G. C. New developments in goblet cell mucus secretion and function. Mucosal Immunol. 8, 712-719 (2015).

47. Specian, R. D. \& Neutra, M. R. Mechanism of rapid mucus secretion in goblet cells stimulated by acetylcholine. J. Cell Biol. 85, 626-640 (1980).

48. Johansson, M. E. Fast renewal of the distal colonic mucus layers by the surface goblet cells as measured by in vivo labeling of mucin glycoproteins. PLOS ONE 7, e41009 (2012).

49. Gustafsson, J. K. et al. An ex vivo method for studying mucus formation, properties, and thickness in human colonic biopsies and mouse small and large intestinal explants. Am. J. Physiol. Gastrointest. Liver Physiol. 302, G430-G438 (2012)

50. Ridley, C. et al. Assembly of the respiratory Mucin MUC5B a new model for a gel-forming Mucin. J. Biol. Chem. 289, 16409-16420 (2014).

51. Von Engelhardt, W. et al. Functional role of bicarbonate in propionate transport across guinea-pig isolated caecum and proximal colon. J. Physiol. 477, 365-371 (1994).
52. Gustafsson, J. K. et al. Bicarbonate and functional CFTR channel are required for proper mucin secretion and link cystic fibrosis with its mucus phenotype. J. Exp. Med. 209, 1263-1272 (2012).

53. Garcia, M. A. S., Yang, N. \& Quinton, P. M. Normal mouse intestinal mucus release requires cystic fibrosis transmembrane regulator-dependent bicarbonate secretion. J. Clin. Invest. 119, 3497-3497 (2009).

54. Cani, P. D. Gut cell metabolism shapes the microbiome. Science 357, 548-549 (2017).

55. Chen, E. Y., Yang, N., Quinton, P. M. \& Chin, W.-C. A new role for bicarbonate in mucus formation. Am. J. Physiol. Lung Cell Mol. Physiol. 299, L542-L549 (2010).

56. Roediger, W. The colonic epithelium in ulcerative colitis: an energy-deficiency disease? Lancet 316, 712-715 (1980).

57. Martens, E. C., Neumann, M. \& Desai, M. S. Interactions of commensal and pathogenic microorganisms with the intestinal mucosal barrier. Nat. Rev. Microbiol. 16, 457-470 (2018)

58. Werlang, C., Cárcarmo-Oyarce, G. \& Ribbeck, K. Engineering mucus to study and influence the microbiome. Nat. Rev. Mater. 4, 134-145 (2019).

59. Cai, R. et al. Interactions of commensal and pathogenic microorganisms with the mucus layer in the colon. Gut Microbes, 11, 680-690 (2020).

60. Wagner, C., Wheeler, K. \& Ribbeck, K. Mucins and their role in shaping the functions of mucus barriers. Annu. Rev. Cell. Dev. Biol. 34, 189-215 (2018).

61. Lee, S. M. et al. Bacterial colonization factors control specificity and stability of the gut microbiota. Nature 501, 426-429 (2013).

62. Cullen, T. et al. Antimicrobial peptide resistance mediates resilience of prominent gut commensals during inflammation. Science 347, 170-175 (2015).

63. Donaldson, G. et al. Gut microbiota utilize immunoglobulin A for mucosal colonization. Science 360, 795-800 (2018).

64. Donaldson, G. P. et al. Spatially distinct physiology of Bacteroides fragilis within the proximal colon of gnotobiotic mice. Nat. Microbiol. 5, 746-756 (2020)

65. Pickard, J. M. et al. Rapid fucosylation of intestinal epithelium sustains host-commensal symbiosis in sickness. Nature 514, 638-641 (2014).

66. Pacheco, A. R. et al. Fucose sensing regulates bacterial intestinal colonization. Nature 492, 113-117 (2012).

67. Ng, K. M. et al. Microbiota-liberated host sugars facilitate post-antibiotic expansion of enteric pathogens. Nature 502, 96-99 (2013).

68. Luo, Q. et al. Enterotoxigenic Escherichia coli secretes a highly conserved mucin-degrading metalloprotease to effectively engage intestinal epithelial cells. Infect. Immun. 82, 509-521 (2014).

69. Sequeira, R. P., McDonald, J. A., Marchesi, J. R. \& Clarke, T. B. Commensal bacteroidetes protect against Klebsiella pneumoniae colonization and transmission through IL-36 signalling. Nat. Microbiol. 5, 304-313 (2020).

70. Etcheverría, A. I., Arroyo, G. H., Perdigon, G. \& Parma, A. E. Escherichia coli with anti-O157: H7 activity isolated from bovine colon. J. Appl. Microbiol. 100 384-389 (2006).

71. Sassone-Corsi, M. et al. Microcins mediate competition among Enterobacteriaceae in the inflamed gut. Nature 540, 280-283 (2016).

72. Rea, M. C. et al. Thuricin CD, a posttranslationally modified bacteriocin with a narrow spectrum of activity against Clostridium difficile. Proc. Natl Acad. Sci. USA 107, 9352-9357 (2010)

73. Rea, M. C. et al. Antimicrobial activity of lacticin 3147 against clinical Clostridium difficile strains. J. Med. Microbiol. 56, 940-946 (2007).

74. Maclntyre, D. L., Miyata, S. T., Kitaoka, M. \& Pukatzki, S. The Vibrio cholerae type VI secretion system displays antimicrobial properties. Proc. Natl Acad. Sci. USA 107, 19520-19524 (2010).

75. Sana, T. G. et al. Salmonella typhimurium utilizes a T6SS-mediated antibacterial weapon to establish in the host gut. Proc. Natl Acad. Sci. USA 113 E5044-E5051 (2016).

76. Chatzidaki-Livanis, M., Geva-Zatorsky, N. \& Comstock, L. E. Bacteroides fragilis type VI secretion systems use novel effector and immunity proteins to antagonize human gut Bacteroidales species. Proc. Natl Acad. Sci. USA 113 3627-3632 (2016).

77. Wheeler, K. M. et al. Mucin glycans attenuate the virulence of Pseudomonas aeruginosa in infection. Nat. Microbiol. 4, 2146-2154 (2019).

78. Wang, B. X. et al. Mucin glycans signal through the sensor kinase RetS to inhibit virulence-associated traits in Pseudomonas aeruginosa. Curr. Biol. 31, 90-102 (2020)

79. Kunzelmann, K. \& Mall, M. Electrolyte transport in the mammalian colon: mechanisms and implications for disease. Physiol. Rev. 82, 245-289 (2002). 
80. Binder, H. J. Role of colonic short-chain fatty acid transport in diarrhea. Annu. Rev. Physiol. 72, 297-313 (2010).

81. Kumar, A. et al. Probiotic Bifidobacterium species stimulate human SLC26A3 gene function and expression in intestinal epithelial cells. Am. J. Physiol. Cell Physiol. 307, C1084-C1092 (2014).

82. Singh, A. K. et al. Tu1846 SLC26A3 (DRA)-deficient mice display dramatically low surface $\mathrm{pH}$, normal mucus secretion but loss of firmly adherent mucus layer, altered colonic microbiome and low grade intestinal inflammation. Gastroenterology 148, 918 (2015).

83. Kini, A. et al. Slc26a3 deletion alters pH-microclimate, mucin biosynthesis, microbiome composition and increases the TNFa expression in murine colon. Acta Physiol., 230, e13498 (2020).

84. Byndloss, M. X. et al. Microbiota-activated PPAR-y signaling inhibits dysbiotic Enterobacteriaceae expansion. Science 357, 570-575 (2017).

85. Leal, J., Smyth, H. D. \& Ghosh, D. Physicochemical properties of mucus and their impact on transmucosal drug delivery. Int. J. Pharm. 532, 555-572 (2017).

86. Swidsinski, A., Loening-Baucke, V., Lochs, H. \& Hale, L. P. Spatial organization of bacterial flora in normal and inflamed intestine: a fluorescence in situ hybridization study in mice. World J. Gastroenterol. 11, 1131 (2005).

87. Swidsinski, A. et al. Viscosity gradient within the mucus layer determines the mucosal barrier function and the spatial organization of the intestinal microbiota. Inflamm. Bowel. Dis. 13, 963-970 (2007).

88. Ijssennagger, N., van der Meer, R. \& van Mil, S. W. Sulfide as a mucus barrier-breaker in inflammatory bowel disease? Trends Mol. Med. 22, 190-199 (2016)

89. Rowan, F. et al. Desulfovibrio bacterial species are increased in ulcerative colitis. Dis. Colon Rectum 53, 1530-1536 (2010).

90. Zinkevich, V. \& Beech, I. B. Screening of sulfate-reducing bacteria in colonoscopy samples from healthy and colitic human gut mucosa. FEMS Microbiol Ecol. 34, 147-155 (2000).

91. Swidsinski, A. et al. Comparative study of the intestinal mucus barrier in normal and inflamed colon. Gut 56, 343-350 (2007).

92. Wiles, T. J. et al. Swimming motility of a gut bacterial symbiont promotes resistance to intestinal expulsion and enhances inflammation. PLoS Biol. 18, e3000661 (2020)

93. Podolsky, D. K., Gerken, G., Eyking, A. \& Cario, E. Colitis-associated variant of TLR2 causes impaired mucosal repair because of TFF3 deficiency. Gastroenterology 137, 209-220 (2009).

94. $\mathrm{Yu}, \mathrm{H}$. et al. The rat IgGFcyBP and Muc2 C-terminal domains and TFF3 in two intestinal mucus layers bind together by covalent interaction. PLOS ONE $\mathbf{6}$, e20334 (2011).

95. Thim, L., Madsen, F. \& Poulsen, S. S. Effect of trefoil factors on the viscoelastic properties of mucus gels. Eur. J. Clin. Invest. 32, 519-527 (2002).

96. Mashimo, H., Wu, D.-C., Podolsky, D. K. \& Fishman, M. C. Impaired defense of intestinal mucosa in mice lacking intestinal trefoil factor. Science $\mathbf{2 7 4}$ 262-265 (1996).

97. Belle, N. M. et al. TFF3 interacts with LINGO2 to regulate EGFR activation for protection against colitis and gastrointestinal helminths. Nat. Commun. 10, 1-13 (2019).

98. Yang, N., Garcia, M. A. S. \& Quinton, P. M. Normal mucus formation requires CAMP-dependent $\mathrm{HCO} 3$ - secretion and $\mathrm{Ca} 2+-$-mediated mucin exocytosis. J. Physiol. 591, 4581-4593 (2013).

99. Yoon, S. S. et al. Anaerobic killing of mucoid Pseudomonas aeruginosa by acidified nitrite derivatives under cystic fibrosis airway conditions. J. Clin Invest. 116, 436-446 (2006).

100. Van der Sluis, M. et al. Muc2-deficient mice spontaneously develop colitis, indicating that MUC2 is critical for colonic protection. Gastroenterology 131, 117-129 (2006).

101. Sommer, F. \& Bäckhed, F. The gut microbiota - masters of host development and physiology. Nat. Rev. Microbiol. 11, 227-238 (2013).

102. Bergstrom, K. et al. Core 1-and 3-derived O-glycans collectively maintain the colonic mucus barrier and protect against spontaneous colitis in mice. Mucosal Immunol. 10, 91-103 (2017).

103. Okayasu, I. et al. A novel method in the induction of reliable experimental acute and chronic ulcerative colitis in mice. Gastroenterology 98, 694-702 (1990).

104. Morampudi, $V$. et al. The goblet cell-derived mediator RELM- $\beta$ drives spontaneous colitis in Muc2-deficient mice by promoting commensal microbial dysbiosis. Mucosal Immunol. 9, 1218-1233 (2016).
105. Bergström, J. H. et al. Gram-positive bacteria are held at a distance in the colon mucus by the lectin-like protein ZG16. Proc. Natl Acad. Sci. USA 113, 13833-13838 (2016).

106. Forman, R. A. et al. The goblet cell is the cellular source of the anti-microbial angiogenin 4 in the large intestine post Trichuris muris infection. PLOS ONE 7, e42248 (2012)

107. Hooper, L. V., Stappenbeck, T. S., Hong, C. V. \& Gordon, J. I. Angiogenins: a new class of microbicidal proteins involved in innate immunity. Nat. Immunol. 4, 269-273 (2003).

108. Okumura, R. et al. Lypd8 inhibits attachment of pathogenic bacteria to colonic epithelia. Mucosal Immunol. 13, 75-85 (2020).

109. Kaetzel, C. S. Cooperativity among secretory IgA, the polymeric immunoglobulin receptor, and the gut microbiota promotes host-microbial mutualism. Immunol. Lett. 162, 10-21 (2014).

110. Dupont, A., Heinbockel, L., Brandenburg, K. \& Hornef, M. W. Antimicrobial peptides and the enteric mucus layer act in concert to protect the intestinal mucosa. Gut microbes 5, 761-765 (2014).

111. Barr, J. J. et al. Bacteriophage adhering to mucus provide a non-host-derived immunity. Proc. Natl Acad. Sci. USA 110, 10771-10776 (2013).

112. Barnich, N. et al. CEACAM6 acts as a receptor for adherent-invasive E. coli, supporting ileal mucosa colonization in Crohn disease. J. Clin. Invest. 117, 1566-1574 (2007).

113. Galtier, M. et al. Bacteriophages targeting adherent invasive Escherichia coli strains as a promising new treatment for Crohn's disease. J. Crohns Colitis 11, 840-847 (2017).

114. Chervy, M., Barnich, N. \& Denizot, J. Adherent-invasive E. coli: update on the lifestyle of a troublemaker in Crohn's disease. Int. J. Mol. Sci. 21, 3734 (2020).

115. Thoma, C. Bacteriophage virome in IBD. Nat. Rev. Gastroenterol. Hepatol. 15, 520-520 (2018).

116. Duerkop, B. A. et al. Murine colitis reveals a disease-associated bacteriophage community. Nat. Microbiol. 3, 1023-1031 (2018).

117. Silveira, C. B. \& Rohwer, F. L. Piggyback-the-Winner in host-associated microbial communities. NPJ Biofilms Microbiomes 2, 1-5 (2016).

118. Gogokhia, L. et al. Expansion of bacteriophages is linked to aggravated intestinal inflammation and colitis. Cell Host Microbe 25, 285-299. e288 (2019).

119. Nishiyama, $K$, et al. Bifidobacterium bifidum extracellular sialidase enhances adhesion to the mucosal surface and supports carbohydrate assimilation. mBio. 8, e00928-17 (2017).

120. Garrido, D., Kim, J. H., German, J. B., Raybould, H. E. \& Mills, D. A. Oligosaccharide binding proteins from Bifidobacterium longum subsp. infantis reveal a preference for host glycans. PLoS ONE 6, e17315 (2011).

121. Troge, A. et al. More than a marine propeller-the flagellum of the probiotic Escherichia coli strain Nissle 1917 is the major adhesin mediating binding to human mucus. Int. J. Med. Microbiol. 302, 304-314 (2012).

122. Owen, C. D. et al. Unravelling the specificity and mechanism of sialic acid recognition by the gut symbiont Ruminococcus gnavus. Nat. Commun. $\mathbf{8}$, $1-15$ (2017).

123. Ho, J. G., Greco, A., Rupnik, M. \& Ng, K. K.S. Crystal structure of receptorbinding C-terminal repeats from Clostridium difficile toxin A. Proc. Natl Acad. Sci. USA 102, 18373-18378 (2005).

124. Ruiz-Palacios, G. M., Cervantes, L. E., Ramos, P., Chavez-Munguia, B. \& Newburg, D. S. Campylobacter jejuni binds intestinal H (O) antigen (Fuca1, 2Gal $31,4 \mathrm{Gl}(\mathrm{NAc})$, and fucosyloligosaccharides of human milk inhibit its binding and infection. J. Biol. Chem. 278, 14112-14120 (2003).

125. Conover, M. S. et al. Inflammation-induced adhesin-receptor interaction provides a fitness advantage to uropathogenic E. coli during chronic infection. Cell Host Microbe 20, 482-492 (2016).

126. Erdem, A. L., Avelino, F., Xicohtencatl-Cortes, J. \& Girón, J. A. Host protein binding and adhesive properties of $\mathrm{H} 6$ and $\mathrm{H} 7$ flagella of attaching and effacing Escherichia coli. J. Bacteriol. 189, 7426-7435 (2007).

127. Dodson, K. W. et al. Structural basis of the interaction of the pyelonephritic $E$. coli adhesin to its human kidney receptor. Cell 105, 733-743 (2001).

128. Buts, L. et al. The fimbrial adhesin F17-G of enterotoxigenic Escherichia coli has an immunoglobulin-like lectin domain that binds N-acetylglucosamine. Mol. Microbiol. 49, 705-715 (2003).

129. Moonens, K. et al. Structural insight in histo-blood group binding by the F18 fimbrial adhesin FedF. Mol. Microbiol. 86, 82-95 (2012).

130. Hung, C. S. et al. Structural basis of tropism of Escherichia coli to the bladder during urinary tract infection. Mol. Microbiol. 44, 903-915 (2002). 
131. Chessa, D., Winter, M. G., Jakomin, M. \& Bäumler, A. J. Salmonella enterica serotype Typhimurium Std fimbriae bind terminal a $(1,2)$ fucose residues in the cecal mucosa. Mol. Microbiol. 71, 864-875 (2009).

132. Mariscotti, J. F., Quereda, J. J., García-del Portillo, F. \& Pucciarelli, M. G. The Listeria monocytogenes LPXTG surface protein Lmo1413 is an invasin with capacity to bind mucin. Int. J. Med. Microbiol. 304 393-404 (2014).
133. Diderrich, R. et al. Structural hot spots determine functional diversity of the Candida glabrata epithelial adhesin family. J. Biol. Chem. 290, 19597-19613 (2015).

134. Henrissat, B. A classification of glycosyl hydrolases based on amino acid sequence similarities. Biochem. J. 280, 309-316 (1991).

135. Ndeh, D. \& Gilbert, H. J. Biochemistry of complex glycan depolymerisation by the human gut microbiota. FEMS Microbiol Rev. 42, 146-164 (2018). 